\title{
qRT-PCR: a method and its difficulties
}

\author{
Franziska Bollmann • Ingrid Casper • Jenny Henke • \\ Andrea Pautz
}

Received: 16 July 2012 / Accepted: 18 July 2012 / Published online: 4 August 2012

(C) Springer-Verlag 2012

Today, quantitative reverse transcription polymerase chain reaction (qRT-PCR) is the favored method to quantify gene expression in molecular biology and clinical studies. qRTPCR analyses can be performed in a high-throughput manner and are very sensitive and specific, cost-effective, and reproducible. Even though this technique is easy to handle, the executer has to pay attention to some critical points. Right from the beginning of proper data generation, it is important to store the samples correctly and to prepare pure and intact RNA. It is, for example, more difficult to obtain good-quality RNA from paraffin-embedded samples than of frozen tissues (Takano et al. 2010). There are RNA protection and preparation kits available that help to maintain the integrity of RNA. Subsequently, an effective reverse transcription reaction (RT) and a specific primer design are indispensable for a high PCR efficiency. For a clear survey, Udvardi's group posted 11 golden rules of performing qRT-PCR analyses and gave a concise support from sample collection to data analysis (Udvardi et al. 2008).

Before performing qRT-PCR analyses, the choice between two detection methods has to be made: nonprobe-based and probe-based assays. Nonprobe-based analyses use an intercalating dye like SYBR ${ }^{\circledR}$ green or ethidium bromide for the target detection. The dye intercalates into all double stranded

Franziska Bollmann, Ingrid Casper, and Jenny Henke contributed equally to this editorial.

\section{F. Bollmann}

Center of Thrombosis and Hemostasis (CTH),

Medical Center of the Johannes Gutenberg University Mainz, Langenbeckstraße 1,

55131 Mainz, Germany

I. Casper $\cdot$ J. Henke $\cdot$ A. Pautz $(\bowtie)$

Department of Pharmacology,

Medical Center of the Johannes Gutenberg University Mainz,

Obere Zahlbacher Strasse 67,

55131 Mainz, Germany

e-mail: pautz@uni-mainz.de
DNAs formed during the PCR reaction. To exclude the detection of unspecific PCR products, it is necessary to analyze the PCR products further by melting curves or gel electrophoresis. The major advantage of nonprobe-based analyses even in high throughput experiments is the lower investment, because each optimized PCR can easily be converted into a quantitative PCR. In contrast, probe-based detection needs an additional oligonucleotide that binds internal to the amplified target sequence and contains a quencher and reporter. The probe has to be designed for every target sequence, and this, in turn, guarantees a higher specificity for the detection system. Several different probe structures can be used for this method: TaqMan ${ }^{\circledR}$, molecular beacons, or Scorpion Primers.

A crucial point of qRT-PCR analyses is the normalization of the results that, improperly done, can have a profound influence on study conclusions (Ferguson et al. 2010). Even though normalization is a highly discussed topic, mostly neither editors nor reviewer demands on the proof of a suitable data evaluation. Current used normalization strategies range from standardization of sample size, like tissue weight or RNA amount, to internal and external standards. When normalizing with external standards, the absolute copy number of the target transcripts can be determined. The standard curve can either be generated from diluted plasmid DNA ( $\mathrm{Li}$ and Wang 2000) or from in vitro-transcribed RNAs (Workenhe et al. 2008). The quantification is based on the assumption that plasmid DNA and cDNA [reverse transcribed from messenger RNA (mRNA)] have the same amplifications rates. In contrast to the external control, it is also possible to use coamplified internal controls. This normalization strategy to one or more internal controls is commonly used (Vandesompele et al. 2002). The principle of this method is based on the quantification of the mRNA of an internal reference gene that undergoes the same procedures and conditions as the mRNA of interest. Using this kind of normalization, it is absolutely essential to search for a reference gene whose expression is 
neither influenced by the experimental conditions (Weiss et al. 2012) nor differently regulated in various organs, tissues, or cell lines that might be of interest (Cabiati et al. 2012). Socalled housekeeping genes (HKGs) are often used as internal controls for qRT-PCR analyses. HKGs are defined to be constitutively expressed in many tissues, organs, and organisms and are essential for cell survival. However, it is already known that commonly used reference genes, like glyceraldehyde-3phosphate dehydrogenase, beta-2-microglobulin, or rRNA, can be regulated in various tissues or under certain experimental conditions (Guenin et al. 2009; Stahlberg et al. 2004). In this issue, Weiss et al. (2012) show a concentration-dependent regulation of several HKGs by rifampicin. They demonstrate that the regulation of those HKGs, used for normalization, leads to misinterpretation of qRT-PCR-data. A first advice, whether a reference gene is regulated by different conditions, in organs, or cell types, may be the $C_{t}$ values measured. The $C_{t}$ values should not differ for more than 0.5 numerical values when conditions like RNA quality and amount, RT efficiency, and the quantity of the performed experiments are considered equally. If reference genes are obviously regulated, microarray analyses can be performed to get an idea of unregulated genes in the experimental settings (Coito et al. 2012). However, it is necessary to confirm the microarray results again by qRT-PCR to verify the $C_{t}$ values. First indications can be given by programs like RefGenes from Genevestigator that compares already demanded microarray data from experiments with similar conditions with the own data (http://www.refgenes.org).

To simplify the search for reference genes programs like GeNorm, BestKeeper or Normfinder have been designed. Those will calculate adequate reference genes, after uploading the data of several experimental conditions. The idea of the often used GeNorm is the pairwise comparison approach of unregulated genes (Vandesompele et al. 2002). It defines the internal control gene-stability measure for every HKG variation and eliminates the worst scoring $\mathrm{HKG}$ in each step. The program lists a ranking of the stability of each HKG. A disadvantage of this knockout method is the elimination of a strong HKG, when compared to another strong HKG. This leads to a possible loss of stable HKGs in early steps. The program BestKeeper is based on a similar analysis, but additionally determines further stably expressed genes. So if BestKeeper determines unregulated target genes, they can be used as an expression standard too (Pfaffl et al. 2004). Normfinder, in contrast, is a model-based approach. It calculates the stability value for all candidate genes and can calculate it for several subgroups like tumor vs. normal tissue. Therefore, the program puts the genes into context and ranks the HKGs due to their "minimal estimated intra- and intergroup variation" (Andersen et al. 2004).

In conclusion, qRT-PCR as a sensitive tool for the determination of mRNA expression requires critical screening of normalization for the experimental outcome. The tissue storage and preparation should be standardized and optimized for each sample. The use of well-established reference genes needs to be tested for every new experimental condition. Several documents, like the MIQE guidelines (Bustin et al. 2009), and computer programs can additionally help to choose the reference genes with the highest stability in the experiment (http://www.gene-quantification.de/). Due to varying reference gene expression, the determination of multiple internal controls or a triplex detection of the RNA of interest and internal and external controls (Vandenbussche et al. 2010) is recommended.

Acknowledgments Work in the authors' lab was supported by the Federal Ministry of Education and Research (BMBF 01EO1003) and grant 961-386261/917 K from the Innovation Foundation of the State of Rhineland-Palatinate.

\section{References}

Andersen CL, Jensen JL, Orntoft TF (2004) Normalization of real-time quantitative reverse transcription-PCR data: a model-based variance estimation approach to identify genes suited for normalization, applied to bladder and colon cancer data sets. Cancer Research 64(15):5245-5250. doi:10.1158/0008-5472.CAN-040496

Bustin SA, Benes V, Garson JA, Hellemans J, Huggett J, Kubista M, Mueller R, Nolan T, Pfaffl MW, Shipley GL, Vandesompele J, Wittwer CT (2009) The MIQE guidelines: minimum information for publication of quantitative real-time PCR experiments. Clinical Chemistry 55(4):611-622. doi:10.1373/clinchem.2008.112797

Cabiati M, Raucci S, Caselli C, Guzzardi MA, D'Amico A, Prescimone T, Giannessi D, Del Ry S (2012) Tissue-specific selection of stable reference genes for real-time PCR normalization in an obese rat model. Journal of Molecular Endocrinology 48 (3):251-260. doi:10.1530/JME-12-0024

Coito JL, Rocheta M, Carvalho L, Amancio S (2012) Microarraybased uncovering reference genes for quantitative real time PCR in grapevine under abiotic stress. BMC Research Notes 5(1):220. doi:10.1186/1756-0500-5-220

Ferguson BS, Nam H, Hopkins RG, Morrison RF (2010) Impact of reference gene selection for target gene normalization on experimental outcome using real-time qRT-PCR in adipocytes. PloS One 5(12):e15208. doi:10.1371/journal.pone.0015208

Guenin S, Mauriat M, Pelloux J, Van Wuytswinkel O, Bellini C, Gutierrez L (2009) Normalization of qRT-PCR data: the necessity of adopting a systematic, experimental conditions-specific, validation of references. Journal of experimental botany 60(2):487493. doi: $10.1093 / \mathrm{jxb} / \mathrm{ern} 305$

Li X, Wang X (2000) Application of real-time polymerase chain reaction for the quantitation of interleukin-1beta mRNA upregulation in brain ischemic tolerance. Brain Research Brain Research Protocols 5(2):211-217

Pfaffl MW, Tichopad A, Prgomet C, Neuvians TP (2004) Determination of stable housekeeping genes, differentially regulated target genes and sample integrity: BestKeeper-Excel-based tool using pair-wise correlations. Biotechnology Letters 26(6):509-515

Stahlberg A, Hakansson J, Xian X, Semb H, Kubista M (2004) Properties of the reverse transcription reaction in mRNA quantification. Clinical Chemistry 50(3):509-515. doi:10.1373/clinchem.2003.026161 
Takano EA, Mikeska T, Dobrovic A, Byrne DJ, Fox SB (2010) A multiplex endpoint RT-PCR assay for quality assessment of RNA extracted from formalin-fixed paraffin-embedded tissues. BMC Biotechnology 10:89. doi:10.1186/1472-6750-10-89

Udvardi MK, Czechowski T, Scheible WR (2008) Eleven golden rules of quantitative RT-PCR. The Plant Cell 20(7):1736-1737. doi:10.1105/tpc. 108.061143

Vandenbussche F, Vandemeulebroucke E, De Clercq K (2010) Simultaneous detection of bluetongue virus RNA, internal control GAPDH mRNA, and external control synthetic RNA by multiplex real-time PCR. Methods in Molecular Biology 630:97-108. doi:10.1007/978-1-60761-629-0 7
Vandesompele J, De Preter K, Pattyn F, Poppe B, Van Roy N, De Paepe A, Speleman F (2002) Accurate normalization of real-time quantitative RT-PCR data by geometric averaging of multiple internal control genes. Genome Biology 3 (7):RESEARCH0034

Weiss J, Theile D, Haefeli WE (2012) Rifampicin alters the expression of reference genes used to normalize real-time quantitative RTPCR data. Naunyn Schmiedebergs Arch Pharmacol this Issue

Workenhe ST, Kibenge MJ, Iwamoto T, Kibenge FS (2008) Absolute quantitation of infectious salmon anaemia virus using different real-time reverse transcription PCR chemistries. Journal of Virological Methods 154(1-2):128-134. doi:10.1016/ j.jviromet.2008.08.007 